\title{
Online removal of ocular artefacts from the electroencephalogram
}

\author{
B.W. Jervis, M.I.B. Garcia, M. Thomlinson and J.M.L. Lopez
}

\begin{abstract}
A method by which ocular artefacts (OAs) in the electroencephalogram (EEG) may be removed automatically online by electro-oculogram (EOG) subtraction is demonstrated. This is achieved by a combination of recursively calculating the required cross-correlations, a fast matrix inversion method, and the use of a modern microprocessor with a high clock rate. Although recursive calculations are involved, the method itself is essentially non-recursive, which means that distortion of any evoked potentials is minimised. The method may be applied simultaneously to any number of EEG channels.
\end{abstract}

\section{Introduction}

The human electroencephalogram (EEG) is frequently contaminated by ocular artefacts (OAs). These are large varying electrical scalp potentials associated with eye movements or blinks, and are caused by movement of a corneo-retinal electric dipole and the short-circuiting of it by the eyelid [1-3]. The change in potential is the ocular artefact, which is superimposed upon the EEG and any evoked potentials (EPs) present. EPs are signals of smaller magnitude, which appear in the EEG in response to certain stimuli. Typically the magnitudes of the signals may be: OA - O $(1000 \mu \mathrm{V})$; EEG - O $(100 \mu \mathrm{V})$; EP - O $(10 \mu \mathrm{V})$. The dipoles also produce electrical potential differences measurable between electrodes placed around the eye, which are known as electro-oculograms (EOGs). Since the EEG and the EPs have clinical applications it becomes necessary to remove the ocular artefacts from EEG recordings. Current clinical practice involves the EEGer inspecting the recordings and recognising the presence of OAs or removing them offline. Computerised online removal of the EEGs would simplify the inspection task and save time by rendering offline removal unnecessary.

Ocular artefact removal (OAR) has been reviewed in [4]. Methods based on modelling the OAs as a sum of EOG terms and then subtracting the modelled OA from the measured EEG to provide an estimate of the artefact-free EEG have been the most widely used. The method may in principle be applied offline using a non-recursive algorithm, or online using a recursive algorithm. In practice, online multi-channel OA removal (OAR) has been restricted by the speed of the available microprocessors to only a few EEG channels, but this restriction has now been overcome with the availability of faster microprocessors.

\footnotetext{
(C) IEE, 2003

IEE Proceedings online no. 20030757

doi:10.1049/ip-smt:20030757

Paper received 22nd May 2003

B.W. Jervis is with the School of Engineering, Sheffield Hallam University, Pond Street, Sheffield S1 1WB, UK

M.I.B. Garcia and J.M.L. Lopez are with the Department of Computer Science, Escuela Universtaria Politecnica, Cordoba, Spain

M. Thomlinson is with the School of Science and Mathematics, Sheffield Hallam University, Pond Street, Sheffield S1 1WB, UK
}

Where online OAR is implemented recursively using earlier methods [5] the shapes of the EPs are modified [6]. Furthermore, because the methods are imperfect some artefact in the corrected EEG will always remain [7], whether it be corrected recursively or non-recursively. It has been established in simulations that after OAR the EEG power remained modified by $3 \%$ over a $14 \mathrm{~s}$ recording, and that the radial component of the OA [8] contributed up to $30 \%$ of the residual OA power [9]. The residual artefact within the EEGs was not visually detectable. Good results in differentiating between subject categories based on EPs have nevertheless been achieved using EOG subtraction $[10,11]$. In [12] an online non-linear recursive method is described. This method may suffer from similar problems to those encountered in the linear recursive technique.

An expert system technique has been applied to identify OAs and to distinguish them from pathological waves in the EEG $[13,14]$. This allowed OAR at the point of occurrence only. Unfortunately, this approach cannot be adopted more widely without the knowledge base and rules.

The purpose of this paper is to show how multi-channel OAR may now be achieved online by EOG subtraction. This is achieved by the combination of a recursive crosscorrelation calculation, a fast matrix inversion, and a higher-speed microprocessor than had hitherto been available. It is shown that the results obtained agree with those of an intensive previous investigation of OAR [9], in which a quantitative theory of residual OA was presented, together with the results of measurements and calculations of residual artefact power. Without being a central purpose of the work comparisons of this method with others have been made [7].

\section{Theory of ocular artefact removal}

In the EOG subtraction method [9, 15] the ocular artefact $\mathbf{O A}$ in an EEG signal is represented by a linear model of the form:

$$
\begin{gathered}
\mathbf{O A} \approx \theta_{\mathbf{1}} \mathbf{E O G}_{\mathbf{1}}+\theta_{2} \mathbf{E O G}_{\mathbf{2}}+\ldots+\theta_{i} \mathbf{E O G}_{\mathbf{i}} \\
+\ldots+\theta_{n} \mathbf{E O G}_{\mathbf{n}}
\end{gathered}
$$

where the $\mathbf{E O G}_{\mathbf{i}}$ are the electro-oculogram signals (represented as a vector of signal samples in which $\mathbf{E O G}_{\mathbf{i}}=\left\{\mathrm{EOG}_{\mathrm{i}, 0}, \quad \mathrm{EOG}_{\mathrm{i}, 1}, \ldots \mathrm{EOG}_{\mathrm{i}, \mathrm{N}-1}\right\}$ for the $\mathrm{N}$ signal samples from 0 to $\mathrm{N}-1$ ) measured between pairs 
of electrodes placed around the eyes, and the $\theta_{i}$ are parameters of the model. The vector, $\mathbf{E E G}_{\mathbf{M}}$, of measured EEG signals containing ocular artefacts can then be represented in terms of $\mathbf{E E G}_{\mathbf{T}}$, the true EEG, as:

$$
\begin{aligned}
\mathbf{E E G}_{\mathbf{M}}= & \mathbf{E E G}_{\mathbf{T}}+\theta_{1} \mathbf{E O G}_{\mathbf{1}}+\theta_{2} \mathbf{E O G}_{\mathbf{2}} \\
& +\ldots+\theta_{i} \mathbf{E O G}_{\mathbf{i}}+\ldots+\theta_{m} \mathbf{E O G}_{m}
\end{aligned}
$$

A common approach [16] to find an estimate, $\mathbf{O A}_{\mathbf{E}}$, of the $\mathbf{O A}$ is to make a least squares fit of the linear model (2) to $\mathbf{E E G}_{\mathbf{M}}$. It is shown in [15] that the least squares estimates for the $\theta_{i}$, are given by the solution of the linear equations:

$$
\hat{\boldsymbol{\theta}}=\left(\mathbf{X}^{\mathbf{T}} \mathbf{X}\right)^{-\mathbf{1}} \mathbf{X}^{\mathbf{T}} \mathbf{E} \mathbf{E} \mathbf{G}_{\mathbf{M}}
$$

where $\hat{\boldsymbol{\theta}}^{\mathrm{T}}=\left[\hat{\theta}_{1}, \hat{\theta}_{2}, \ldots . \hat{\theta}_{m}\right]$ is the vector of parameter estimates $\hat{\boldsymbol{\theta}}$ for a model of order $\mathrm{m}, \mathbf{X}$ is the matrix whose $\mathrm{jth}$ column is the column vector $\mathbf{E O G}_{\mathbf{j}},\left(\mathbf{X}^{\mathrm{T}} \mathbf{X}\right)$ is a matrix of cross-correlations at zero lag of the EOG ${ }_{i S}$, and $\mathbf{X}^{\mathbf{T}} \mathbf{E E} \mathbf{G}_{\mathbf{M}}$ is a matrix of cross-correlations of $\mathrm{EOG}_{\mathrm{iS}}$ and $\mathrm{EEG}_{\mathrm{M}} \mathrm{s}$. The mean values of the signals are removed either during or before the correlation calculations. $\mathbf{E E G}_{\mathbf{E}}$, the estimated EEG, is taken as the remaining signal after the estimated artefact has been subtracted from the measured EEG, thus:

$$
\begin{aligned}
\mathbf{E E G}_{\mathbf{E}}= & \mathbf{E E G}_{\mathbf{M}}-\left(\hat{\theta}_{1} \mathbf{E O G}_{\mathbf{1}}+\hat{\theta}_{2} \mathbf{E O G}_{\mathbf{2}}\right. \\
& \left.+\ldots+\hat{\theta}_{i} \mathbf{E O G}_{\mathbf{i}}+\ldots+\hat{\theta}_{m} \mathbf{E O G}_{m}\right)
\end{aligned}
$$

\section{Implementation}

The aim was to perform online OAR. It is shown here how OAR may be implemented online not by a recursive algorithm, but by using a recursion in the computation (which accelerates the computation of the matrix) and a faster microprocessor. Writing (3) in full for one EEG channel gives:

$$
\begin{aligned}
&\left(\begin{array}{lll}
\sum x_{1}^{2}(i) & \sum x_{1}(i) x_{2}(i) & \sum x_{1}(i) x_{3}(i) \ldots \\
\sum x_{2}(i) x_{1}(i) & \sum x_{2}^{2}(i) & \sum x_{2}(i) x_{3}(i) \ldots \\
\vdots & \vdots & \vdots \\
\sum m_{m}(i) x_{1}(i) & \sum x_{m}(i) x_{2}(i) & \sum x_{m}(i) x_{3}(i) \ldots \\
& \ldots \sum x_{1}(i) x_{m}(i) \\
& \ldots \sum x_{2}(i) x_{m}(i) \\
& \vdots \\
& \ldots \sum x_{m}^{2}(i)
\end{array}\right)\left(\begin{array}{l}
\hat{\theta}_{1} \\
\hat{\theta}_{2} \\
\vdots \\
\hat{\theta}_{m}
\end{array}\right) \\
&=\left(\begin{array}{l}
\sum x_{1}(i) y(i) \\
\sum x_{2}(i) y(i) \\
\vdots \\
\sum x_{m}(i) y(i)
\end{array}\right)
\end{aligned}
$$

where the summations are carried out over $i=0$ to $i=N-1$, i.e. over the $\mathrm{N}$ sample points, and $m$ is the model order (= number of EOG terms in the OA model).

It is seen that all the terms on the right hand side of (5) are $\mathrm{N}$ times the cross-correlations at zero lag of either the EOGs, or of the EOGs by the EEG. The matrix to be inverted is also symmetrical about the leading diagonal, and therefore contains $\frac{m}{2}(m+1)$ different cross-correlations. The total number of cross-correlations in (5) is $\frac{m}{2}(m+1)+m$. For $M$ EOG channels there are $\frac{m}{2}(m+1)+$ $m M$ different correlations. Each correlation involves the computation of $N$ products and $N-1$ sums. Altogether for $M$ EOG channels, therefore, $\left(\frac{m}{2}(m+1)+m M\right) N$ products and $\left(\frac{m}{2}(m+1)+m M\right)(N-1)$ sums must be calculated. For typical values of $m=4, M=32$, and $N=250$ there are 33,500 products and 33,366 sums. These must all be executed within one sampling interval if on-line OAR is to be feasible. For signals sampled at $125 \mathrm{~Hz}$, the sampling interval will be $8 \mathrm{~ms}$. Thus, we sought to reduce the number of computations.

Consider the data to be viewed through a sliding window of length $n$ points with the final point in the window being indexed as $i$. The cross-correlations were speeded up using the recursion [17]:

$$
R_{a b}^{i}(0)=R_{a b}^{i-1}(0)+a(i) b(i)-a(i-n) b(i-n)
$$

where $R_{a b}^{i}(0)$ is the zero lag cross-correlation evaluated for the points $i$ in the sequences $a(i)$ and $b(i)$ of length $n$. When the computation begins the windows contain $n-1$ zeros, correct cross-correlations being obtained after the first $n-1$ cross-correlations have been computed. Each cross-correlation now only involves two multiplications and two sums (one negative). With $n=250$, this represents an increase in speed by a factor of 125 .

The EOG subtraction method requires that the DC levels of the voltages be set to zero for good results [16]. This must be effected for each data window and is also, therefore, most efficiently done recursively for any sequence a(i) using:

$$
\bar{a}(i)=\bar{a}(i-1)+\frac{1}{n}(a(i)-(a(i-n))
$$

It is possible to combine the cross-correlations and the DC level removal in one recursive algorithm. Let the average of any sequence $a(i)$ be denoted as:

$$
\bar{a}(i)=\frac{1}{n} \sum_{i=0}^{n-1} a(i)
$$

The cross-correlation function is:

$$
R_{a b}^{i}(0)=\sum_{i=0}^{n-1} a(i) b(i)
$$

When the DC levels have been removed the crosscorrelation function becomes:

$$
R_{a b, j}^{i, D C}(0)=\sum_{i=0}^{n-1}(a(i)-\bar{a}(i))(b(i)-\bar{b}(i))
$$

which upon expansion simplifies to:

$$
R_{a b}^{i, D C}(0)=R_{a b}^{i}(0)-n \bar{a}(i) \bar{b}(i)
$$

Substituting (6) into (11) gives:

$$
R_{a b}^{i}(0)=R_{a b}^{i-1}(0)+a(i) b(i)-a(i-n) b(i-n)
$$

and

$$
R_{a b}^{i, D C}(0)=R_{a b}^{i}(0)-n \bar{a}(i) \bar{b}(i)
$$

which together with equations similar to (7) for $\bar{a}(i)$ and $\bar{b}(i)$ constitutes the recursive algorithm.

Given all the values of the cross-correlations, (5) is calculated for each EEG channel to obtain the model parameters $\theta$ for each of the points $i$. Thus, the $\theta$ are computed adaptively for each window. Equation (4) is then used to obtain the estimated EEG at $i$.

The solution of the linear equations (5) once per sampling interval was achieved using the method of $\mathbf{L U}$ decomposition [18].

\section{Method}

A number of EEGs in the absence of eye movements and blinks were recorded as well as EEGs and four EOGs in the 
presence of OAs as described in [9]. EOG voltages $\mathrm{V}_{1}, \mathrm{~V}_{2}$, $\mathrm{V}_{3}$, and $\mathrm{V}_{4}$ were measured (Fig. 1). The vertical right EOG, $\mathrm{VEOG}_{\mathrm{R}}$, the horizontal right EOG, $\mathrm{HEOG}_{\mathrm{R}}$, the horizontal left EOG, $\mathrm{HEOG}_{\mathrm{L}}$, and the radial right EOG, $\mathrm{REOG}_{\mathrm{R}}$, were then given by:

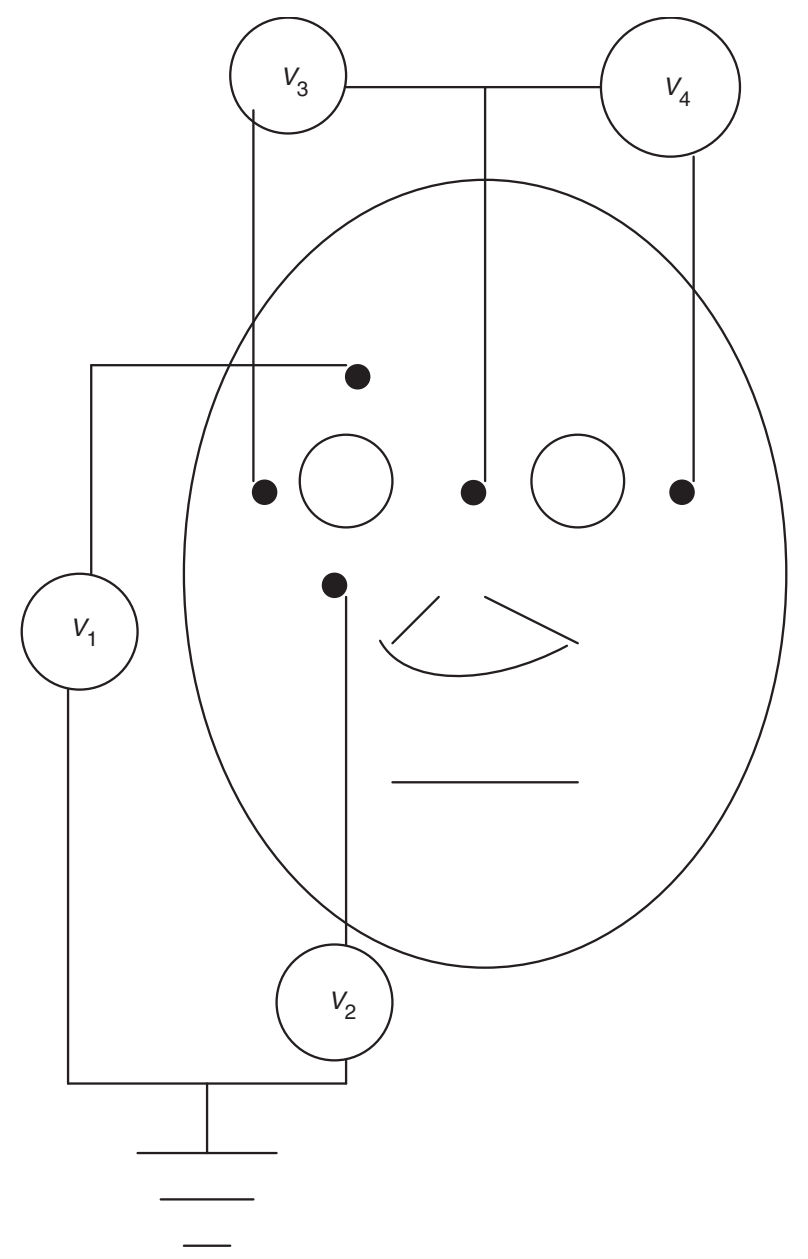

Fig. 1 EOG electrode sites and measured voltages

$\mathrm{VEOG}_{\mathrm{R}}=\mathrm{V}_{1}-\mathrm{V}_{2}, \quad \mathrm{HEOG}_{\mathrm{R}}=\mathrm{V}_{3}, \quad \mathrm{HEOG}_{\mathrm{L}}=\mathrm{V}_{4}$, and $\mathrm{REOG}_{\mathrm{R}}=\left(\mathrm{V}_{1}+\mathrm{V}_{2}\right) / 2$. EEGs were measured between electrode sites $\mathrm{C}_{\mathrm{Z}}, \mathrm{P}_{4}, \mathrm{~F}_{4}, \mathrm{O}_{1}$ and linked mastoids. Quasiperiodic vertical, horizontal, and oblique eye movements, and also random eye movements with blinks, generated corresponding EOGs. The sampling frequency was $125 \mathrm{~Hz}$.

OA contaminated measured EEGs were also simulated by adding an OA model (1) to EEG measured $\left(\mathrm{EEG}_{\mathrm{M}}\right)$ with stationary eyes (2). One model consisted of $V_{E O G}$, $\mathrm{HEOG}_{\mathrm{R}}$, and $\mathrm{HEOG}_{\mathrm{L}}$; the second also included REOG $\mathrm{R}$. Model coefficients in the range $\theta=0.2$ to 0.7 were used to introduce significant amounts of artefact. Recorded OAcontaminated $\mathrm{EEG}_{\mathrm{M}} \mathrm{S}$ were also processed. Comparisons were made between this method and the non-recursive OAR.

The time needed to correct 8 EEG recordings of 14 seconds length were measured using twelve different window lengths between $n=10$ points and $n=512$ points. From these, the time to correct the current 32 points corresponding to 32 EEG channels within one sampling period of $8 \mathrm{~ms}$ was calculated. The processor clock frequency was $150 \mathrm{Mz}$. With modern faster processors operating at, as an example, $1.2 \mathrm{GHz}$, the computation time is reducible by a factor of 8 , or alternatively 256 channels could be corrected in the same time.

\section{Results and discussion}

\subsection{Residual artefact}

The powers $\mathrm{POA}_{\mathrm{R}}, \mathrm{PEEG}_{\mathrm{E}}$, and $\mathrm{POA}_{\mathrm{T}}$ of the voltage waveforms of $\mathrm{OA}_{\mathrm{R}}$ (the residual $\mathrm{OA}$ voltage), $\mathrm{EEG}_{\mathrm{E}}$ (the estimated $\mathrm{EEG}$ voltage), and $\mathrm{OA}_{\mathrm{T}}$ (the total $\mathrm{OA}$ voltage), respectively, were calculated for the $14 \mathrm{~s}$ recordings. Note that $\mathrm{EEG}_{\mathrm{E}}=\mathrm{EEG}_{\mathrm{M}}-\mathrm{OA}(\mathrm{OA}$ model$)$, and $\mathrm{OA}_{\mathrm{R}}=$ $E_{E} G_{E}-E E G_{T}$. In this way, example power ratios of $\mathrm{POA}_{\mathrm{R}} / \mathrm{PEEG}_{\mathrm{E}}$ were found to be $2.3 \%$ (vertical EM), $9 \%$ (horizontal and oblique EMs), 10\% (blinks), and 19.6\% (random EMs). The ratio of $\mathrm{POA}_{\mathrm{R}} / \mathrm{POA}_{\mathrm{T}}$ lay between $5.8 \%$ and $20 \%$. These results were in general agreement with those in [9], suggesting that the method functioned correctly. Example $\mathrm{EEG}_{\mathrm{T}}, \mathrm{EEG}_{\mathrm{E}}$, and $\mathrm{OA}_{\mathrm{R}}$ waveforms are shown in Fig. 2.

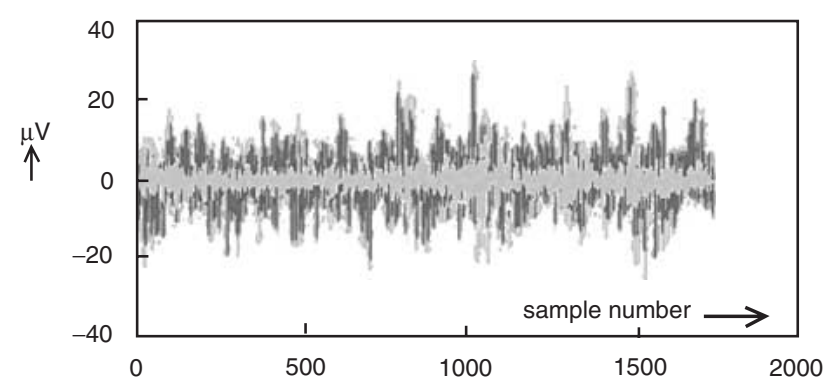

Fig. $2 E E G_{T}$ (largest amplitude trace), $E E G_{E}$, and $O A_{R}$ (smallest amplitude trace)

\subsection{Timings}

The time required to correct the current sample from each of 32 channels for different window lengths was determined. With $\mathrm{n}=200$ this was $8.05 \mathrm{~ms}$, which just exceeded the sampling interval and also resulted in some distortion. With $\mathrm{n}=400$ (no distortion) the time was $15.8 \mathrm{~ms}$, just under two sampling intervals. Thus, with a $150 \mathrm{MHz}$ clock, 16 channels of EEG can be corrected, and with a $1.2 \mathrm{GHz}$ clock, 128 channels can be corrected online. More channels could be accommodated by using more microprocessors.

\subsection{Real data}

In Fig. 3 the corrected EEGs are plotted for the case of quasi-periodic vertical eye movements, with and without including the radial component of the electrical dipole. Incorporation of the radial component resulted in more complete OAR.

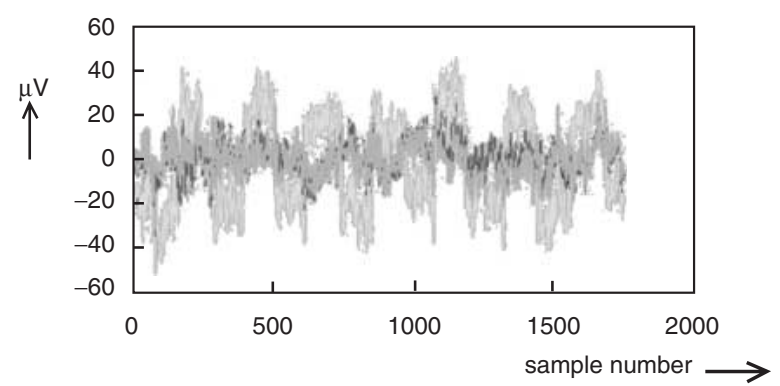

Fig. 3 The measured $E E G_{T}$ (largest amplitude trace) and the corrected EEGs for the case of quasi-periodic vertical eye movements with (smallest amplitude trace) and without including the radial component of the electrical dipole 


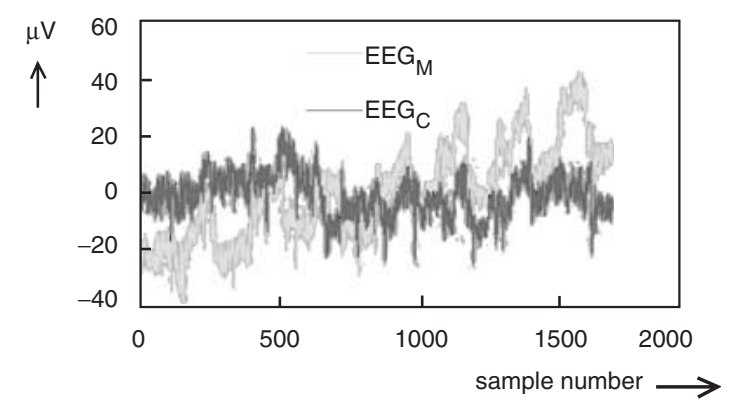

Fig. 4 Measured and corrected EEGs in the presence of vertical $E M$

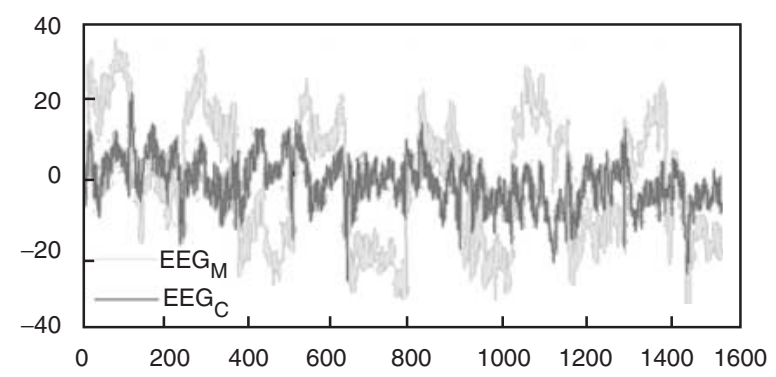

Fig. 5 Measured and corrected EEGs in the presence of oblique $E M$

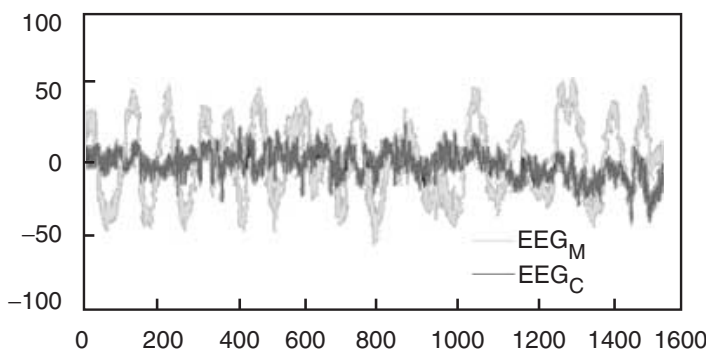

Fig. 6 Measured and corrected EEGs in the presence of random $E M$

In Fig. 4 the measured and corrected EEGs in the presence of vertical EM are shown. Corresponding waveforms are plotted in Figs. 5 and 6 for oblique and random EMs, respectively. The gross artefact can be seen to have been removed in each case. In Fig. 7 the EEGs are shown for online OAR using the algorithm and for the offline non-recursive method in the case of random eye movements. There is some divergence in the waveforms at later sample numbers.

\subsection{Remarks}

Previously suggested online OAR methods were recursive [5] and caused distortion of evoked potentials in the EEG [6]. In this method, each voltage sample is corrected nonrecursively such that the distortion is avoided. Furthermore, the number of computations, and hence the time needed to correct the corresponding time samples from all the different channels, is considerably reduced by performing the associated cross-correlations for each temporally succeeding set of samples recursively. The method may be described as adaptive and non-recursive in time.

The results given here are in agreement with the quantitative results for the amounts of residual artefact in the EEGs corrected by EOG subtraction as given elsewhere
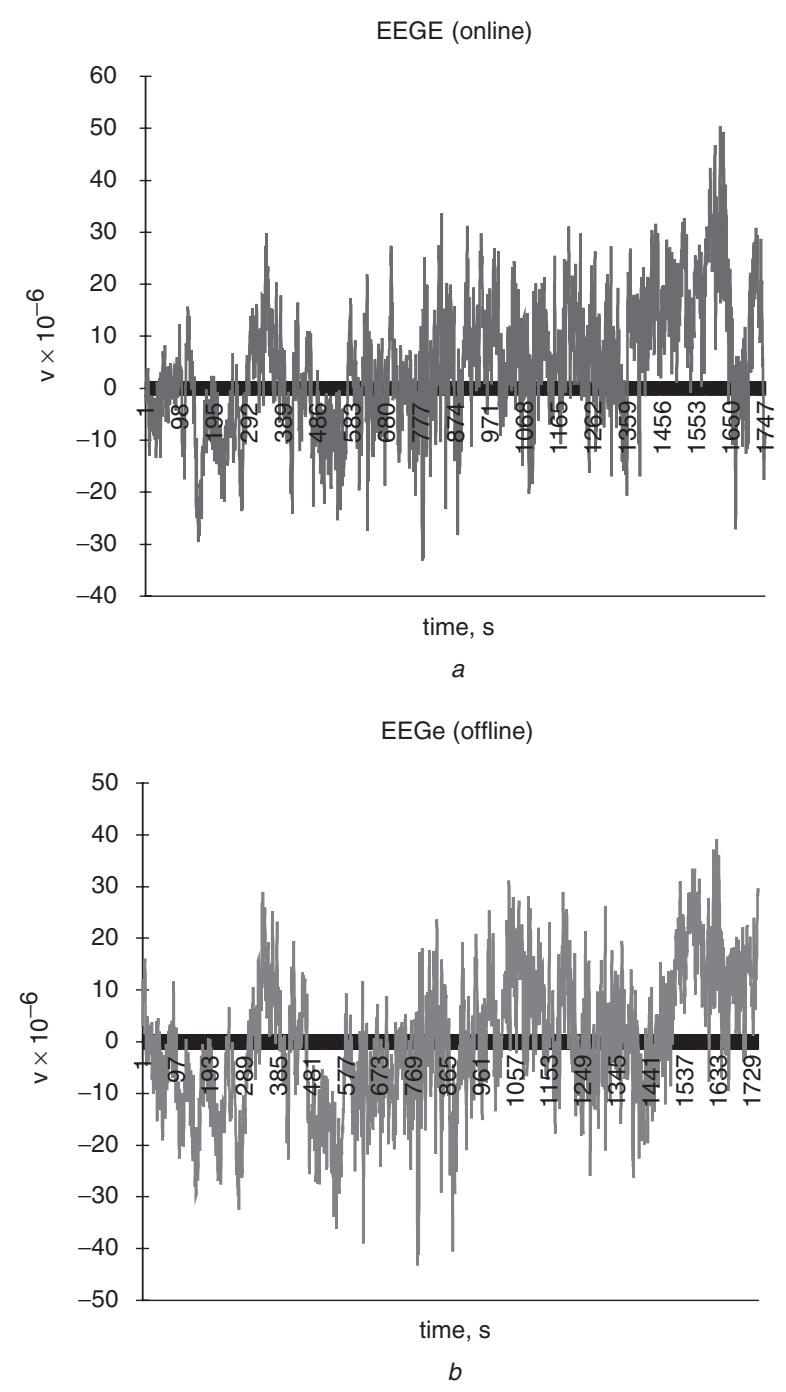

Fig. 7 Estimated EEG calculated $a$ online $b$ offline

[9]. Reference is made to that paper for full details and for the theory of residual OA.

\section{Conclusions}

It has been demonstrated that using modern microprocessors with clock rates of about $1 \mathrm{GHz}$ ocular artefacts in multi-channel EEGs can be removed online using the EOG subtraction method.

\section{Acknowledgement}

M. I. B. Garcia and J. M. L. Lopez thank the ERASMUS scheme for enabling them to work in Professor Jervis' research group at Sheffield Hallam Universitiy.

\section{References}

1 Hillyard, S.A.: 'Methodological issues in CNV research', in Thompson, R.F. and Patterson, M.M. (Eds.) 'Bioelectric recording techniques part B, Electroencephalography and human brain potentials' (Academic Press, 1974), pp. 259-280

2 Matsuo, F., Peters, J.F., and Reilly, E.I.: 'Electrical phenomena associated with movements of the eyelid', Electroencephalogr. Clin. Neurophysiol., 1975, 38, pp. 507-511

3 Barry, W., and Jones, G.M.: 'Influence of eyelid movement upon electro-oculographic recording of vertical eye movements', Aerospace Medicine, 1965, 36, pp. 855-858 
4 Jervis, B.W., Ifeachor, E.C., and Allen, E.M.: 'The removal of ocular artefacts from the electroencephalogram: a review', Med. Biol. Eng. Comput., 1988, 26, pp. 2-12

5 Ifeachor, E.C., Jervis, B.W., Morris, E.L., Allen, E.M., and Hudson, N.R.: 'A new microcomputer-based online ocular artefact removal (OAR) system', IEE Proc., A. Phys. Sci. Meas. Instrum. Manage. Educ. Rev., 1986, 133, (5), pp. 291-300

6 Jervis, B.W., Coelho, M., and Morgan, G.W.: 'Effect on EEG responses of removing ocular artefacts by proportional EOG subtraction', Med. Biol. Eng. Comput., 1989, 27, pp. $484-490$

7 Brunia, C.H.M., Möcks, J., Berg-Lenssen, M.M.C., Coelho, M., Coles, M.G.H., Elbert, T., Gasser, T., Gratton, G., Ifeachor, E.C., Jervis, B.W., Lutzenberger, W., Sroka, L., van Blokland-Vogelesang, A.W., van Driel, G., Woestenburg, J.C., Berg, P., McCallum, W.C., Tuan, P.D., Pocock, P.V., and Roth, W.T.: 'Correcting ocular artifacts in the EEG: a comparison of several methods', J. Psychology, 1989, 3, pp. $1-50$

8 Elbert, T., Lutzenberger, W., Rockstroh, B., and Birmbaumer, N 'Removal of ocular artefacts from the EEG: a biophysical approach to the EOG', Electroencephalogr. Clin. Neurophysiol, 1985, 60, pp. 455-463

9 Jervis, B.W., Thomlinson, M., Mair, C., Lopez, J.M.L., and Garcia, M.I.B.: 'Residual ocular artefact subsequent to ocular artefact removal from the electroencephalogram', IEE Proc., Sci. Meas. Technol., 1999, 146, (6), pp. 293-298

10 Jervis, B.W Saatchi, M. R Lacey, A Roberts, T Allen, E.M Hudson, N.R., Oke, S., and Grimsley, M.: 'Artificial neural network and spectrum analysis methods for detecting brain diseases from the CNV response in the electroencephalogram', IEE Proc. A, Science, Meas Technol, 1994, 141, (6), pp. 432-440
11 Jervis, B.W., Garcia, T., and Giahnakis, E.P.: 'Rapid clinical classification by the Probabilistic Simplified Fuzzy ARTMAP'. Proc 3rd Int. Conf. on Neural networks and expert systems in medicine and healthcare (NNESMED), Pisa, Italy, 2-4 September 1998, pp. 205-216

12 Rao, K.D., and Reddy, D.C.: 'On-line method for enhancement of electroencephalogram signals in presence of electro-oculogram artefacts using nonlinear recursive least squares technique', Med. Biol. Eng. Comput., 1995, 33, pp. 488-491

13 Ifeachor, E.C., Hellyar, M.T., Mapps, D.J., and Allen, E.M.: 'Knowledge-based enhancement of human EEG signals', IEE Proc. F, Radar Signal Process., 1990, 137, (5), pp. 302-310

14 Hellyar, M.Y., Ifeachor, E.C., Mapps, D.J., Allen, E.M., and Hudson, N.R.: 'Expert System approach to electroencephalogram signal processing', Knowl. Based Syst., 1995, 8, (4), pp. 164-173

15 Ifeachor, E.C., Jervis, B.W., Allen, E.M., Morris, E.L., Wright, D.E. and Hudson, N.R. 'Investigation and comparison of some models for removing ocular artefacts from EEG signals, Part 1 Review of models and data analysis', Med. Biol. Eng. Comput., 1988, 26, pp. 584-590

16 Jervis, B.W., Coelho, M., and Morgan, G.W.: 'Effect on EEG responses of removing ocular artefacts by proportional EOG subtraction', Med. Biol. Eng. Comput., 1989, 27, pp. 484-490

17 Jervis, B.W., Goude, A., Thomlinson, M., Mir, S., and Miller, G. 'Least squares artefact removal by transputer'. Presented at IEE Colloquium on The transputer and signal processing, 5 March, 1990, London

18 Stroud, K.A.: 'Further Engineering Mathematics' (Macmillan, 1996, 3rd edn.) 